\title{
Rapid and precise diagnosis of pneumonia coinfected by Pneumocystis jirovecii and Aspergillus fumigatus assisted by next-generation sequencing in a patient with systemic lupus erythematosus: a case report
}

Yili Chen

Sun Yat-Sen University

Lu Ai

Sun Yat-Sen University

Yingqun Zhou

First Peoples Hospital of Nanning

Yating Zhao

Sun Yat-Sen University

Jianyu Huang

Sun Yat-Sen University

Wen Tang

Sun Yat-Sen University

Yujian Liang ( $\sim$ lyujian@mail.sysu.edu.cn )

Sun Yat-Sen University

\section{Case report}

Keywords: Pneumocystis jirovecii, Aspergillus fumigatus, Next generation sequencing, Case report

Posted Date: March 19th, 2021

DOI: https://doi.org/10.21203/rs.3.rs-154016/v2

License: (a) (1) This work is licensed under a Creative Commons Attribution 4.0 International License.

Read Full License 


\section{Abstract}

Background: Pneumocystis jirovecii and Aspergillus fumigatus, are opportunistic pathogenic fungus that has a major impact on mortality in patients with systemic lupus erythematosus. With the potential to invade multiple organs, early and accurate diagnosis is essential to the survival of SLE patients, establishing an early diagnosis of the infection, especially coinfection by Pneumocystis jirovecii and Aspergillus fumigatus, still remains a great challenge.

Case presentation: In this case, we reported that the application of next

-generation sequencing in diagnosing Pneumocystis jirovecii and Aspergillus fumigatus coinfection in a Chinese girl with systemic lupus erythematosus (SLE). Voriconazole was used to treat pulmonary aspergillosis, besides sulfamethoxazole and trimethoprim (SMZ-TMP), and caspofungin acetate to treat Pneumocystis jirovecii infection for 6 days. On Day 10 of admission, her chest radiograph displayed obvious absorption of bilateral lung inflammation though the circumstance of repeated fever had not improved. Unfortunately, the patient discharged from the hospital since the financial burden, therefore further condition couldn't be tracked.

Conclusions: This successful application of the next generation sequencing assisting the rapid diagnosis of Pneumocystis jirovecii and Aspergillus fumigatus coinfection provides a new perspective in the clinical approach against the systematic fungi infections and highlights the potential of this technique in rapid etiological diagnosis.

\section{Background}

Systemic lupus erythematosus (SLE) is a chronic multi-systemic autoimmune disease that involve any organ of the body, and infection remains an important cause of morbidity and mortality in patients with SLE [1]. The incidence of fungal infection, especially Aspergillus infection, has increased significantly in patients with SLE, which has become one of the main causes of death in critically ill patients with SLE $[2,3]$. Pneumocystis jiroveciiis, the causative agent of Pneumocystis pneumonia (PCP), which is a common and often life-threatening opportunistic infection in both HIV-infected and non- HIV immunocompromised patients. Compared with the HIV-infected population, the number of the non-HIV immunocompromised patients including SLE patients with PCP is much higher, and the in-hospital mortality reaches from $24 \%$ to $67 \%[4,5,6,7,8]$.

Early diagnosis and appropriate treatment is essential to the survival of SLE patients, but establishing an early diagnosis of the infection, especially coinfection by Pneumocystis jirovecii and Aspergillus fumigatus, still remains a great challenge [9]. Pneumocystis coinfections with other microorganisms are less frequently described and only sparse reports of combined PCP and Aspergillus fumigatus infections exist in the literature, especially in the non- HIV patients [10]. On the other hand, coinfections of 
Pneumocystis jirovecii and other microorganisms are considered as index of poor prognosis in immunocompromised patients, especially when there is invasive aspergillosis [11]. Under the circumstances, a revolutionary technology called next-generation sequencing (NGS) emerged in 2005 has become an attractive alternative method for broad-based pathogen discovery due to the rapid turnaround time and high accuracy. In this manuscript, we described an interesting case of pulmonary coinfection by Pneumocystis jirovecii and Aspergillus fumigatus which was rapid diagnosed by next-generation sequencing within 24 hours in SLE patients, and to our knowledge it is the first documented case in SLE patients.

\section{Case Presentation}

A 14-year-old girl was admitted to hospital (The First Affiliated Hospital of Sun Yat-sen University,

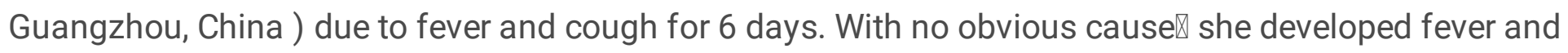
occasional dry cough without dyspnea and headache. Four months ago, she presented to the pediatric ward for edema and foam urine, and further examination demonstrated that antinuclear antibodies (ANA) $181.65 \mathrm{U} / \mathrm{ml}$ (normal range, 0-12 U/ml), anti-double-stranded DNA antibody (dsDNA) $643.19 \mathrm{lU} / \mathrm{ml}$ (normal range, 0-30 IU/ml), Complement $30.12 \mathrm{~g} / \mathrm{L}$ (normal range, 0.79-1.17 g/L), Complement 4 0.03g/L (normal range, 0.17-0.31 g/L), skin rash, urine sugar and protein positive, which made the diagnosed of systemic lupus erythematosus (SLE) established according to American College of Rheumatology (ACR) criteria. She was administered combined methylprednisolone $60 \mathrm{mg} / \mathrm{d}$, hydroxychloroquine, and immunosuppressant tacrolimus.

On admission (Day 1$)$, she got hyperthermia $\left(38.8^{\circ} \mathrm{C}\right)$. Upon physical examination, her blood pressure was $129 / 84 \mathrm{mmHg}$, pulse rate $96 /$ minute and respiratory rate $27 /$ minute. It was characterized by hemorrhagic spots and purple streaks scattered all over the skin, and depressed edema of both lower extremities. Initial laboratory evaluation revealed leukocytosis $\left(17.50 \times 10^{9} / \mathrm{L}, 86 \%\right.$ neutrophils) with high serum Creactive protein $(36.83 \mathrm{mg} / \mathrm{L}$, normal range $0-10 \mathrm{mg} / \mathrm{L})$. Platelet count was normal $\left(189 \times 10^{9} / \mathrm{L}\right)$. Elevate IL6 level was $21.43 \mathrm{pg} / \mathrm{mL}$ (normal range $0-5.3 \mathrm{pg} / \mathrm{mL}$ ) with procalcitonin level at $0.23 \mathrm{mg} / \mathrm{L}$ (normal range 0-0.05mg/L). Arterial blood gases were: p02 $58.7 \mathrm{mmHg}, \mathrm{pCO} 236.8 \mathrm{mmHg}, \mathrm{sO} 293.4 \%$ and pH 7.45 . The additional tests revealed the plasma glucose $12.1 \mathrm{mmol} / \mathrm{L}$ (normal range $3.9-6 \mathrm{mmol} / \mathrm{L}$ ), 2 hours plasma glucose after oral glucose tolerance test (OGTT) $15.1 \mathrm{mmol} / \mathrm{L}$ (normal range $<7.8 \mathrm{mmol} / \mathrm{L}$ ), the glycated hemoglobin of $9.90 \%$ (normal range $4.4-6.4 \%$ ) and urine glucose positive, which suggested the diagnosis of diabetes.

Initial antibiotherapy with intravenous drip of meropenem $1.0 \mathrm{~g}$ every $8 \mathrm{~h}$ was started. However, the oxygenation of the patient continued to deteriorate, with recurrent fever. On Day 4 the chest computed tomography (CT) imaging revealed: diffuse ground glass changes in the bilateral lungs, partial 
consolidation and mild bronchiectasis of the anterior basal ganglia in the right lower lobe, and bullae formation in apical segment of upper lobe of left lung (Figure 1). Without any clinical respiratory improvement, another microbiological tests were performed. Serum $\beta-(1,3)$-D glucan was elevated (436.1 $\mathrm{pg} / \mathrm{mL}$ ) with galactomannan (GM) level at 0.4 (cutoff value of 0.90 ).

On day 5, bonchoalveolar lavage fluid (BALF) were obtained for microbiological examination. Next generation sequencing the BALF specimen was performed, which identified numerous Aspergillus fumigatus nucleotide sequences ( 8,833 of $22,887,333$ cover rate $0.038 \%$ ) and Pneumocystis jirovecii $(109,593$ of $22,887,333$, cover rate $0.48 \%$ ) within 24 hours (Table 1$)$. Initial antibiotherapy with intravenous drip of meropenem $1.0 \mathrm{~g}$ every $8 \mathrm{~h}$ was started. However, the oxygenation of the patient continued to deteriorate, with recurrent fever. Contemporary inspection results show that BALF galactomannan (GM) was strong positive (4.90, cutoff value of 0.90$)$, and the hyphaes was detected with rapid fluorescence detection as Aspergillus (Figure 2a). Moreover, we performed hexamine silver staining on the BALF and it demonstrated positive confirming the existence of pneumocystis infection(Figure 2b). According to the EORTC/MSG definition [12], based on host factors, clinical features, and microbiological evidence冈this patient was diagnosed as the probable invasive pulmonary aspergillosis (IPA). Therefore on Day 5, treatment against coinfection of Pneumocystis jirovecii and Aspergillus fumigatus was started. Antibiotic therapy was changed to voriconazole to treat pulmonary aspergillosis, besides sulfamethoxazole and trimethoprim (SMZ-TMP), and caspofungin acetate to treat Pneumocystis jirovecii infection. Hormone methylprednisolone was reduced to $20 \mathrm{mg}$ per day, and hydroxychloroquine was discontinued. On Day 8, Aspergillus fumigatus was isolated on Sabouraud's agar. On Day 10, chest radiograph displayed obvious absorption of bilateral lung inflammation(Figure 3), though the recurrent of fever. Unfortunately, considering the severity of the patient's condition, difficulty in immune reconstitution and poor prognosis, the patient discharged from the hospital, since her family chose abandonment of treatment due to financial burden. Therefore further condition couldn't be tracked.

\section{Discussion And Conclusion}

Systemic lupus erythematosus (SLE) is a chronic autoimmune disease involving multiple organs and system. Immune system disorder, T cell and B cell dysfunction, the use of corticosteroids and immunosuppressive agents result in low immunity and prone to infection in SLE patients. At the mean time, infection can also aggravate the activity of SLE [13]. The infection site in SLE patients are widely distributed, and the most common infection sites were the lungs, followed by upper respiratory tracts and urinary tracts [14]. A retrospective review performed in 3,831 hospitalized SLE patients revealed that in SLE death cases mainly dying from infection, Aspergillus fumigatus and Pneumocystis carinii were the two most commonly infected pathogens, and Cytomegalovirus was a frequent pathogen of polyinfection [15]. It was worth noting that polyinfections were more frequent than single pathogen infection (60.5 vs. $39.5 \%$ ), including common bacteria, fungal infection, and cytomegalovirus, simultaneously the mortality was significantly higher.The mix infections caused by Aspergillus fumigatus as well as Pneumocystis 
jirovecii are serious and often lethal diseases in severely immunocompromised patients [16]. Moreover the incidence of (IPA) and (PCP) increases with the extent of the underlying or therapeutic immunosuppression, and, especially for IPA, has a major impact on morbidity and mortality [17].

An enhancement of clinical awareness $\ a$ rapid and accurate diagnostic $\mathbb{}$ and precise management is crucial for early treatment in immunocompromised SLE patients. However in severely immunocompromised patients, simultaneous or secondary infections in Pneumocystis jirovecii pneumonia are mostly caused by bacterial pathogens, whereas fungal pathogens, especially Aspergillus fumigatus, are rarely diagnosed [18]. Nowadays the diagnosis of Pneumocystis pneumonia (PCP) is usually achieved by examining stained smears of BALF for the presence of the organism, and the cloning and sequencing of Pneumocystis jiroveciiis provide an alternative method. Moreover, the diagnosis of IPA relies mainly on tissue culture and morphological analysis, which may bring drawbacks due to the limited culture positive rate and is sometimes time consuming [19]. The introduction of biomarkers such as galactomannan (GM) and polymerase chain reaction (PCR) assays make big difference in the diagnosis of invasive pulmonary aspergillosis (IPA) [20]. With advantages of high sensitivity, low cost and fast detection, however, PCR method requires the physician to raise a few suspicious pathogens prior to examination and is usually restricted to only limited range of pathogens. The radiographic evaluation still has difficulty to make the radiographic diagnosis of combined IPA and PCP. Therefore, these methods are not best applicable in certain clinical situations such as polyinfection in SLE patients.

Next-generation sequencing (NGS) or massively parallel sequencing, a method of simultaneously sequencing millions of fragments of DNA, has been rapidly adopted in the clinical laboratory. Under such circumstances, the implementation of NGS in clinical field enabled a fast and comparably accurate diagnostic tool for physicians,and most importantly, it does not require a predefined range of suspicious pathogens.NGS is an assay that can sequence the entire DNA/RNA of a sample, and this process does not need any primers or probes. It holds the promise of identifying most of the pathogens. Furthermore, NGS can generate billions of DNA/RNA sequences per run, and this enables metagenomic analysis. The principles of NGS in infectious diseases are composed of three main procedures, the identification of the nucleotides in the targeted samples, the comparison of these nucleotides against the catalogue of causative agents, and the decision-making progress whether the acquired sequences points to the possible etiological hypothesis [21]. The first literature applying NGS in the diagnosis of infection was published in 2014, in which leptospira sequence were detected in the spinal fluid of neuroleptospirosis patient [22]. Since then, multiple studies have reported the use of NGS in central nervous system, bloodstream, and respiratory infections [23-26]. The advantage of NGS is its potential to detect multiple pathogens simultaneously in one test while immunosuppressed patients are more likely to be the largest beneficiaries of NGS because they often have coinfections [27]. The major challenge of clinical use of NGS is how to interpret the results and determine whether the microorganism whose sequences are 
identified is truly the causative pathogen. The nature of NGS tends to detect all nucleotide sequences not only from the samples but also those acquired from the contamination during clinical procedures or laboratory processing, and thus it is challenging to discriminate causative pathogens from normal microbes and environmental contaminants. A microbe found by NGS is usually considered a causative agent based in part or in total on the following clues or evidence: the coverage rate (species level) is significantly greater than that of any other microbe or that in the control materials, or the mapping read number is at the top of the microbes list [28]; the result is confirmed by traditional techniques such as culture, serological testing, PCR, Sanger sequencing, and histopathology; the sequence or read number or pathogen load is significantly reduced during the course of treatment [29]; and the patient improved after targeted antimicrobial agent treatment $[28,30,31]$.

Detecting specific microorganisms by NGS is just the first step. However, not all microorganisms determined by NGS are relevant to infectious diseases, such as oral colonizing bacteria (e.g., Streptococcus parasanguinis, Streptococcus mitis, and Prevotella melaninogenica). Taken together, clinicians should identify the real pathogen according to both NGS and other examinations.

In our case, NGS identified Pneumocystis jirovecii and Aspergillus fumigatus the causative agent within 24 hours. The sequencing data, which was in consistent with the patient's biological and morphologic evidence, clinical and radiological features, assisted clinical physicians in approaching the diagnosis of mixed infection apace and effectively. With the help of NGS on the rapid diagnosis, the patient's chest radiograph displayed obvious absorption of bilateral lung inflammation after 6 days of target treatment. The limitation of this case study included lacking a validated quantification method to interpret the significance of the NGS result, and since the patient discharged from the hospital, her further condition couldn't be tracked.

In summary, we report a case of a SLE patient with simultaneous Pneumocystis pneumonia and invasive aspergillosis, and as we know it is the first documented case in SLE patients. This case highlights the challenges in the diagnosis and management of this rare dual infection and some of its devastating consequences. The introduction of next-generation sequencing applied to pathogen identification dramatically improves the ability to identify poly-infection that occasionally cause disease but lethal. It helps clinicians to make a rapid and precise diagnosis of infectious diseases in the near future.

\section{Declarations}

\section{Abbreviations}

SLE, Systemic lupus erythematosus 
PCP, Pneumocystis pneumonia

IPA, Invasive Pulmonary Aspergillosis

NGS, next-generation sequencing

BALF, Broncho alveolar lavage fluid

EORTC/MSG,the European Organization for Research and Treatment of Cancer and the Mycoses Study Group.

\section{Ethics approval and consent to participate}

This report was approved by the Clinical Research and Ethics Committee of the First Affiliated Hospital of Sun Yat-sen University.

\section{Consent for publication}

Written informed consent was obtained from the patient's legal guardian for publication of this case report and any accompanying images. A copy of the written consent is available for review by the Editorin-Chief of this journal.

\section{Availability of Data and Materials}

Data and materials of this report are publicly available.

\section{Competing Interest}

The authors declare that they have no competing interests

\section{Funding}

None

\section{Authors Contributions}

YLC and YJL traced the case, and were responsible for the laboratory examinations and writing the manuscript. WT interpreted the patient data regarding the infection disease. LA and YQZ participated in the design and organizing the materials. YTZ and JYH participated in strain collection and literature searching. All authors read and approved the final manuscript.

\section{Acknowledgements}

None 


\section{References}

[1] Dubula T, Mody GM (2015) Spectrum of infections and outcome

among hospitalized South Africans with systemic lupus erythematosus. Clin Rheumatol 34(3):479-488

[2] Wu L, Wang X, Chen F, et al. (2017) T cell subsets and immunoglobulin G levels are associated with the infection status of systemic lupus erythematosus patients. Braz J Med Biol Res 51(2):e4547

[3] Jung JY, Suh CH (2017) Infection in systemic lupus erythematosus, similarities, and differences with lupus flare. Korean J Intern Med 32(3):429-438.

[4] Morris A, Norris KA. Colonization by Pneumocystis jirovecii and its role in disease. Clin Microbiol Rev. 2012;25:297-317.

[5] Matsumura Y, Shindo Y, linuma Y et al. Clinical characteristics of Pneumocystis pneumonia in nonHIV patients and prognostic factors including microbiological genotypes. BMC Infect Dis. 2011;11:76.

[6] Monnet X, Vidal-Petiot E, Osman D et al.Critical care management and outcome of severe Pneumocystis pneumonia in patients with and without HIV infection. Crit Care. 2008;12:R28.

[7] Pereira-Diaz E, et al. Changing Trends in the Epidemiology and Risk Factors of Pneumocystis Pneumonia in Spain. Front Public Health. 2019;7:275. doi:10.3389/fpubh.2019.00275.

[8] Maini R, et al . Emerg Infect Dis.2013);19:386-92. doi:10.3201/eid1903.121151

[9] Rashid R, Denning DW: Invasive pulmonary aspergillosis 10 years post bone marrow transplantation: A case report. J Med Case Rep 2009;3:26.

[10] Baumann S, Reinwald M, Haghi D et al. Coinfection of Pneumocystis

jirovecii and invasive pulmonary aspergillosis in an immunocompromised patient: a diagnostic challenge. Onkologie. 2013;36:582-584.

[11] Anthi-Marina Markantonatou, Aliki loakimidou, Kostoula Arvaniti et al. Pulmonary co- infections by Pneumocystis jiroveciiand Aspergillus fumigatusin non- HIV patients: A report of two cases and literature review. Mycoses. 2017;60:626-633.

[12] J. Peter Donnelly et al.Revision and Update of the Consensus Definitions of Invasive Fungal Disease From the European Organization for Research and Treatment of Cancer and the Mycoses Study Group Education and Research Consortium.Clin. Infect. Dis. 2019 Dec 05; DOI:10.1093/cid/ciz1008.

[13] Chengcheng Hou, Ou Jin, Xi Zhang. Clinical characteristics and risk factors of infections in patients with systemic lupus erythematosus.Clinical Rheumatology(2018) 37:2699-2705. 
[14] Jeong SJ, Choi H, Lee HS, Han SH, Chin BS, Baek JH, Kim CO,Choi JY, Song YG, Kim JM (2009) Incidence and risk factors of infection in a single cohort of 110 adults with systemic lupus erythematosus. Scand J Infect Dis 41(4):268-274.

[15] Yunyun Fei, Xiaochun Shi, Fengying Gan, et al. Death causes and pathogens analysis of systemic lupus erythematosus during the past 26 years. Clin Rheumatol (2014) 33:57-63.

[16] Walsh TJ, Anaissie EJ, Denning DW, et al: Treatment of aspergillosis: Clinical practice guidelines of the Infectious Diseases Society Of America. Clin Infect Dis 2008;46:327-360.

[17] Gilroy SA, Bennett NJ: Pneumocystis pneumonia. Semin Respir Crit Care Med 2011;32:775-782.

[18] Neumann S, Krause SW, Maschmeyer G, Schiel X, von Lilienfeld-Toal M: Primary prophylaxis of bacterial infections and Pneumocystis jirovecii pneumonia in patients with hematological malignancies and solid tumors: Guidelines of the Infectious Diseases Working Party (AGIHO) of the German Society Of Hematology And Oncology (DGHO). Ann Hematol 2013;92:433-442.

[19] Lecuit M, Eloit M. The diagnosis of infectious diseases by whole genome next generation sequencing: a new era is opening. Front Cell Infect Microbiol. 2014;4:25

[20] Asai N, Motojima S, Ohkuni Y, Matsunuma R, Nakashima K, Iwasaki T, Nakashita T, Otsuka Y, Kaneko $\mathrm{N}$ : Early diagnosis and treatment are crucial for the survival of Pneumocystis pneumonia patients without human immunodeficiency virus infection. J Infect Chemother 2012;18:898-905.

[21] Jacob HJ. Next-generation sequencing for clinical diagnostics. N Engl J Med. 2013;369(16):1557-8.

[22] Wilson MR, Naccache SN, Samayoa E, et al. Actionable diagnosis of neuroleptospirosis by nextgeneration sequencing. N Engl J Med. 2014;370(25):2408-17.

[23] Grumaz S, Stevens P, Grumaz C, et al. Next-generation sequencing diagnostics of bacteremia in septic patients. Genome Med. 2016;8(1):73.

[24] Kawada J, Okuno Y, Torii Y, et al. Identification of viruses in cases of pediatric acute encephalitis and encephalopathy using next-generation sequencing. Sci Rep. 2016;6:33452.

[25] Cordey S, Brito F, Vu DL, et al. Astrovirus VA1 identified by next-generation sequencing in a nasopharyngeal specimen of a febrile Tanzanian child with acute respiratory disease of unknown etiology. Emerg Microbes Infec. 2016;5(9):e99.

[26] Thorburn F, Bennett S, Modha S, et al. The use of next generation sequencing in the diagnosis and typing of respiratory infections. J Clin Virol. 2015;69:96-100.

[27] Zhang Y, Ai J, Cui P, Zhang W, Wu H, Ye M. 2019. A cluster of cases of pneumocystis pneumonia identified by shotgun metagenomics approach. J Infection. 78(2):158-169. 
[28] Fan S, Qiao X, Liu L, Wu H, Zhou J, Sun R, Chen Q, Huang Y,Mao C, Yuan J, et al. 2018. Nextgeneration sequencing of cerebrospinal fluid for the diagnosis of neurocysticercosis. Front Neurol. 9:471.

[29]Ye M, Wei W, Yang Z, Li Y, Cheng S, Wang K, Zhou T, Sun J,Liu S, Ni N, et al. 2015. Rapid diagnosis of Propionibacterium acnes infection in patient with hyperpyrexia after hematopoietic stem cell transplantation bynext-generation sequencing: a case report. BMC Infect Dis.16(1):5.

[30] Cervera R, Khamashta MA, Font J, et al. Morbidity and mortality in systemic lupus erythematosus during a 5-year period. A multicenter prospective study of 1000 patients. European Working Party on Systemic Lupus Erythematosus [ J ]. Medicine, 1999, 78(3): 167- 175.

[31] Mok CC, Lee KW, Ho CT, et al. A prospective study of survival and pro gnostic indicators of systemic lupus erythematosus in a southern Chinese population [ J] . Rheumatology (Oxford), 2000, 39(4): 399406.

\section{Tables}

\begin{tabular}{|llll|}
\hline \multicolumn{3}{|l|}{ Table1.NGS report of the microorganism in BALF } \\
\hline Genus & Species & \\
\hline Name & Sequence numbera & Name & Sequence numbera \\
\hline Pneumocystis & 110174 & Pneumocystis jirovecii & 109593 \\
\hline Aspergillus & 8891 & Aspergillus fumigatus & 8833 \\
\hline
\end{tabular}

a:The sequence number of the strict comparison of the microorganism detected at the level of genus/species.

\section{Figures}




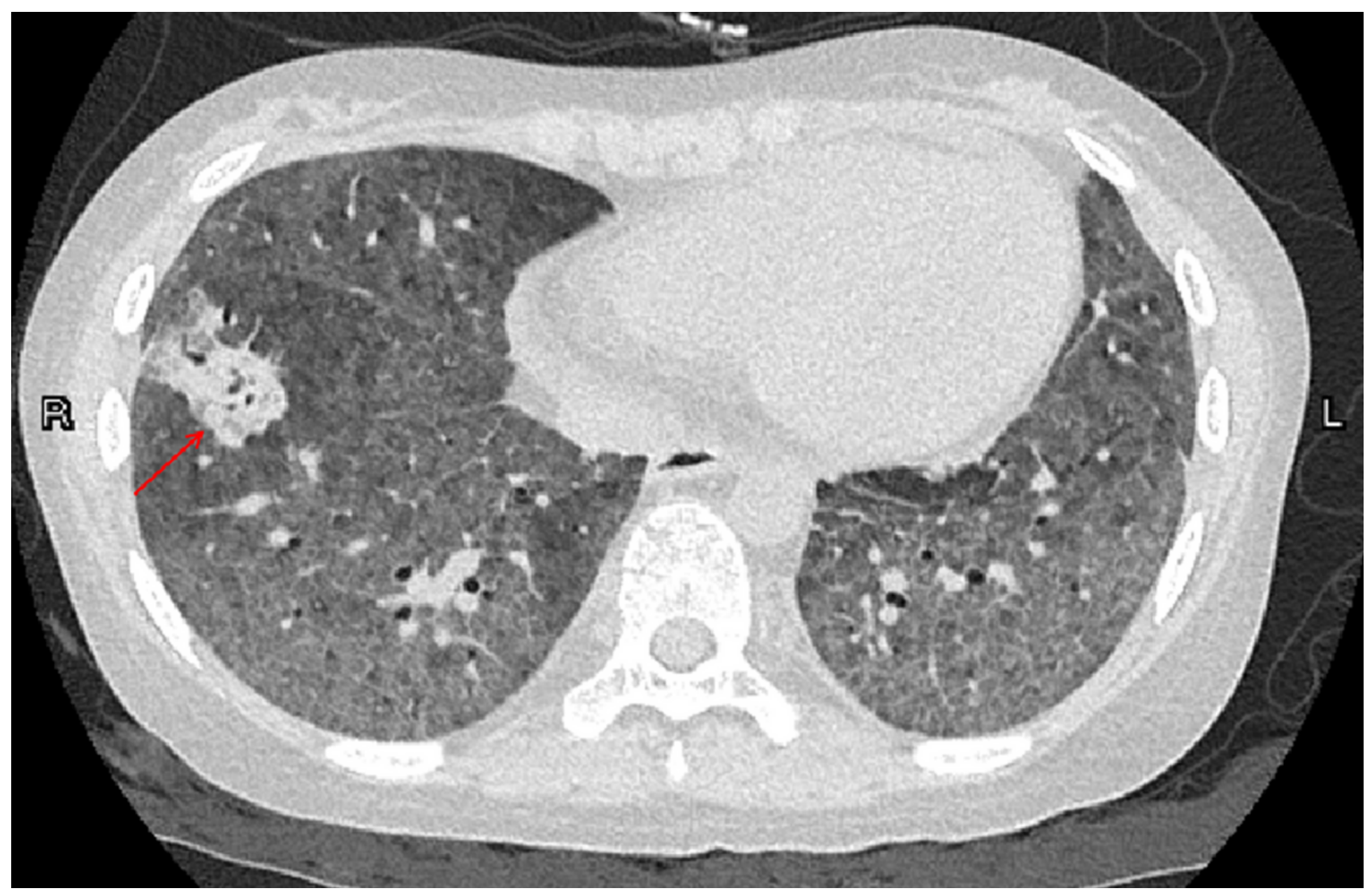

Figure 1

The chest computed tomography (CT) imaging revealed diffuse ground glass changes in the bilateral lungs, partial consolidation(red arrow) and mild bronchiectasis of the anterior basal ganglia in the right lower lobe, and bullae formation in apical segment of upper lobe of left lung.

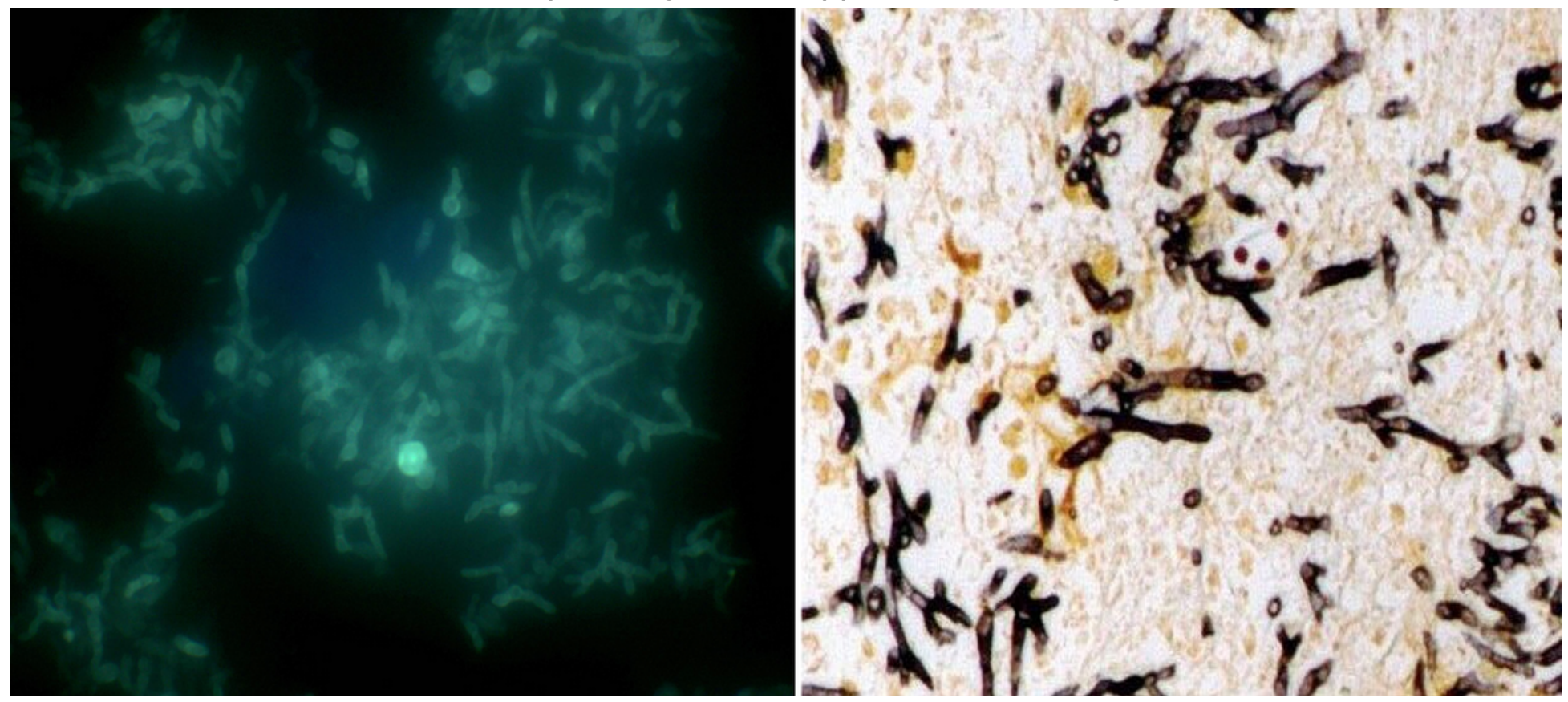




\section{Figure 2}

The hyphaes was detected with rapid fluorescence detection of Aspergillus (2a), and hexamine silver staining on the BALF demonstrated positive (2b).
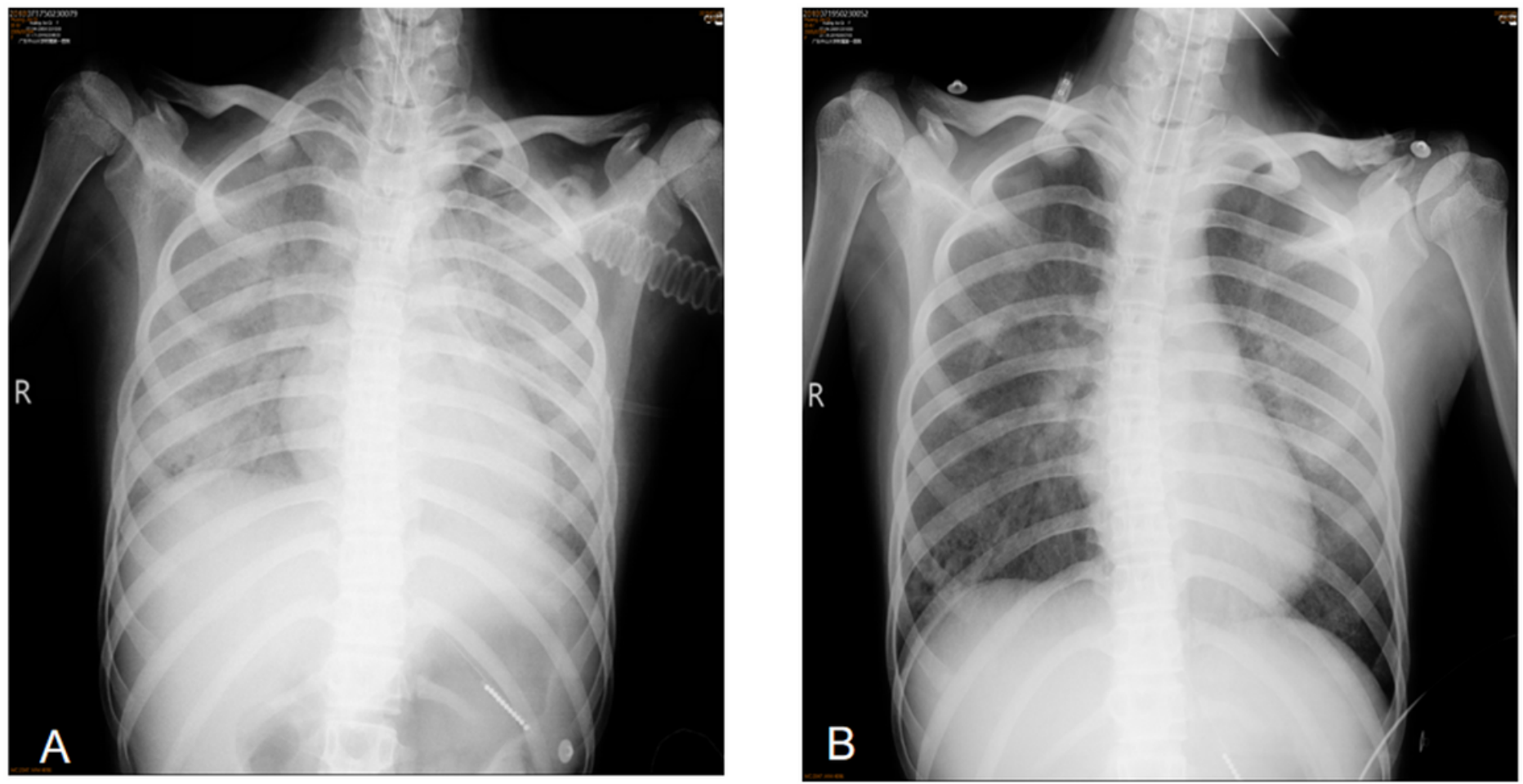

\section{Figure 3}

Compared with the $\mathrm{X}$-ray image on $\operatorname{admission} \operatorname{day}(\mathrm{A})$, the chest radiograph image displayed obvious absorption of bilateral lung inflammation after treatment against Pneumocystis jirovecii and Aspergillus fumigatus(B). 